\title{
A New Perspective on Understanding the Reduced Spring Dust Storm Frequency in Inner Mongolia, China
}

\author{
Ning $\mathrm{Li}^{1,2} \cdot \mathrm{Li} \mathrm{Guo}^{3} \cdot$ Bihang Fan ${ }^{1}$
}

Published online: 21 September 2015

(C) The Author(s) 2015. This article is published with open access at Springerlink.com

\begin{abstract}
Spatiotemporal patterns of dust storms are affected by climate change through changes in convective instability, regional meteorological characteristics, and local sediment supply. Linking dust storm dynamics to climate change helps the understanding of what controls the initiation of dust storms, and assists the prediction of future dust storm occurrence. This study examines the temporal dynamics of spring dust storms in Inner Mongolia, a major dust source area in East Asia. We found that severe spring dust storms have significantly declined from 1954 to 2007. Four dust storm types showed similar decreasing trends from 2001 to 2012 . This change in spring dust storm dynamics is attributed to the shift in vegetation green-up dates based on the analysis of a satellite derived vegetation index. Earlier vegetation green-up has a dampening effect on spring dust storms. Suitable environmental conditions for vegetation green-up hinder the emergence of dust storms. This study expands our understanding of the dynamics of spring dust storms in the changing climate through a new perspective on vegetation phenology in the spring.
\end{abstract}

Ning Li

ningli@bnu.edu.cn

1 State Key Laboratory of Earth Surface Processes and Resource Ecology, Beijing Normal University, Beijing 100875, China

2 Key Laboratory of Environmental Change and Natural Disasters, Ministry of Education, Beijing Normal University, Beijing 100875, China

3 College of Global Change and Earth System Science, Beijing Normal University, Beijing 100875, China
Keywords Climate change $\cdot$ Dust storm dynamics · Green-up date · Inner Mongolia · Vegetation phenology

\section{Introduction}

Dust storms occur in association with strong turbulent winds and exposed soil surfaces (Goudie 1983, 2009) and have wide-ranging implications for the natural system and human society (Goudie and Middleton 1992). East Asia (China, Mongolia, Japan, and the Korean Peninsula) accounts for one fifth of the world's population and includes large areas of arid and semiarid lands. These are some of the strongest source areas for dust storms and pose annual dust storm threats (Goudie and Middleton 1992; Liu et al. 2011).

Dust storms transport mineral nutrients from deserts and drylands to rain forests and oceans, providing bioavailable iron to photosynthetic microorganisms and supporting local ecosystems (Griffin and Kellogg 2004). Dust storm deposits form the fertile loess soils in northern China and the Midwestern US and were reclaimed as agricultural lands (Catt 1988); they became the cradle of Chinese culture. Dust storms also exert strong impacts on local and global climate by modifying radiative forcing, cloud physical properties, and biogeochemical cycling (Idso 1974; Creamean et al. 2013).

Dust storms impact socioeconomic systems significantly, including agricultural productivity, public health, mass transportation, and livelihoods (Schlesinger et al. 1990; Toon 2003). A single dust storm event that occurred on 5 May 1993 threatened 1.1 million $\mathrm{km}^{2}$ of territory in China and resulted in a direct economic loss of CNY 560 million Yuan (about USD 70 million) (Wang et al. 1995). Understanding the controls and spatiotemporal dynamics of 
dust storms has become a growing concern for both scientific research and policy making (Fan et al. 2014).

This article reviews the primary controls of dust storm initiation and examines the long-term trends and variations of spring dust storms in Inner Mongolia-a major dust storm area with a local supply of source materials in East Asia. Two datasets of dust storm field observations were analyzed - severe dust storms from 1954 to 2007; and four dust storm types from 2001 to 2012. After a brief summary of the possible causes of these dust storm dynamics as reported in previous studies, we propose a new perspective to explain the variation of spring dust storms by linking dust storm dynamics to the shift of vegetation phenology in the spring. This study provides a novel view to understanding the variation of dust storms in a changing climate.

\section{Primary Controls of Dust Storm Outbreaks}

Atmospheric circulation systems, near-surface meteorological elements, and underlying surface conditions are the most influential controls of initiating dust storms (Goudie
1983; Qian et al. 2002; Wang et al. 2004; Shao and Dong 2006) (Fig. 1). Atmospheric thermodynamic instabilities determine the strength of turbulences and provide the climate conditions that are favorable for dust storm development (Yang et al. 2008; Li et al. 2014). For example, spring cold fronts from Siberia undercut warm air, the pressure gradient then increases, and winds become gusty in East Asia (Ebata and Tateishi 2001). The strong winds whip up dust materials, eventually forming dust storms (Qian et al. 2002). The convective instabilities make spring a dust storm prevailing season in East Asia (Wang et al. 2004; Shao and Dong 2006). Regional atmospheric conditions play an important role in determining the size, duration, and intensity of dust storms (Zhang et al. 1997; Qian et al. 2002; Shao and Dong 2006).

Local near-surface meteorological elements and underlying surface conditions also contribute to the emergence of dust storms by affecting the access to dust materials (Shao and Dong 2006). Sand composition on the land surface is the material foundation of the formation of a dust storm (Lee and Sohn 2011). A dry or less-precipitation climate tends to create the conditions for the initiation of

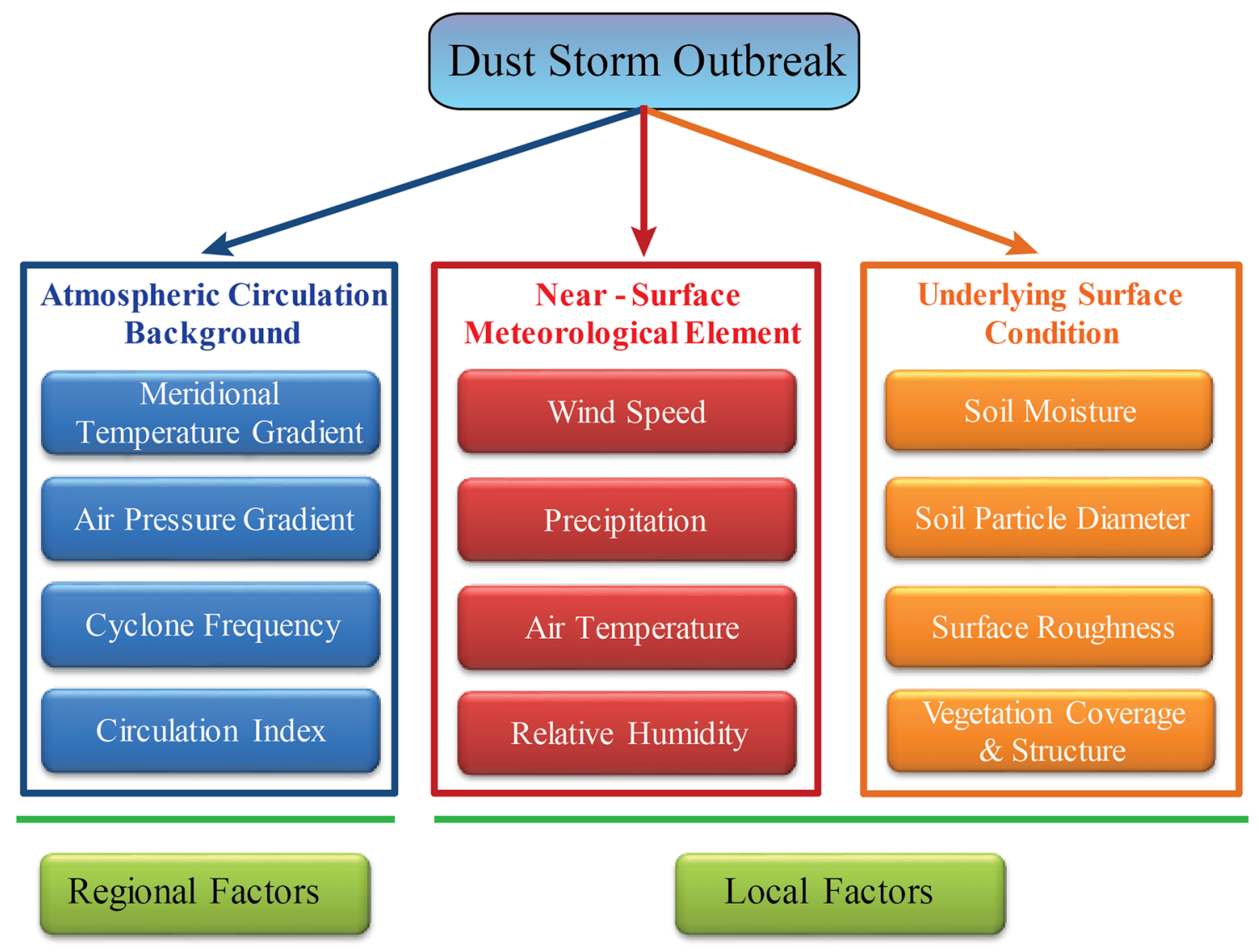

Fig. 1 Primary controls of dust storm initiation. At the regional scale, convective instability provides the driving force of dust storm outbreaks. Locally, near-surface meteorological conditions and underlying surface conditions determine the availability of dust materials, the speed of surface friction wind, and the threshold wind speed for blowing up dust materials 
dust storms (Qian et al. 2002) and dust storms tend to appear in dry places with an annual precipitation of 25-300 mm (Xu and $\mathrm{Hu}$ 1997). In an arid or semiarid climate, the loosely held soil surface particles increase susceptibility of the area to dust storms (Cowie et al. 2013). A greater surface roughness (such as higher vegetation coverage) is likely to slow down the friction velocity and restrain the occurrence of dust storms (Mao et al. 2013). At the same time, soil moisture, soil particle size, and the stability of the underlying surface together determine the threshold wind speed needed to blow up dust materials and affect the frequency of dust storm outbreaks (Song et al. 2005; Kurosaki et al. 2011).

Along with the varying climate, regional atmospheric conditions and local meteorological elements are changing as well, and climate change-induced variations in surface conditions also occur. The pattern and variability of dust storms are inevitably affected by the changing climate (Sun et al. 2001; Fan et al. 2014). Linking dust storm dynamics to climate change not only helps the comprehension of the physical mechanisms that control dust storm outbreaks, but also assists the prediction of dust storm emergence and their impacts on social-ecological systems and people's livelihoods (Schlesinger et al. 1990; Toon 2003; Creamean et al. 2013).

\section{Temporal Variation of Spring Dust Storms in Inner Mongolia in Recent Decades}

Fluctuations of dust storm activity vary at the global scale (Goudie 2009; Shao et al. 2013). Among the three major dust storm source regions-North Africa, the Middle East, and East Asia (Chen 2010; Creamean et al. 2013), an increasing trend of dust storm outbreaks has been observed in North Africa (for example, the Sahel zone) and the Middle East (Goudie and Middleton 1992). East Asia (including the Taklimakan Desert, Northeastern China, and Mongolia) has shown a declining trend in dust storm frequency since the late twentieth century, except for a spike in dust storm activity in 2001 and 2002 (Zhou et al. 2001; Qian et al. 2002; Wang et al. 2004; Shao and Dong 2006). Using the dust storm intensity index calculated from 186 stations in China, Tan et al. (2014) supported the decreasing trend of dust storm days from 1980 to 2008.

To examine the long-term dynamics of spring dust storms in northern China, we analyzed the observations of severe dust storms from 23 meteorological stations in Inner Mongolia (37.61 $\left.-46.78^{\circ} \mathrm{N}, 105.24^{\circ}-119.89^{\circ} \mathrm{E}\right)$ (Fig. 2) over 54 years, from 1954 to 2007 . This severe dust storm dataset is maintained by the China Meteorological Data Sharing Service System, and can be downloaded from

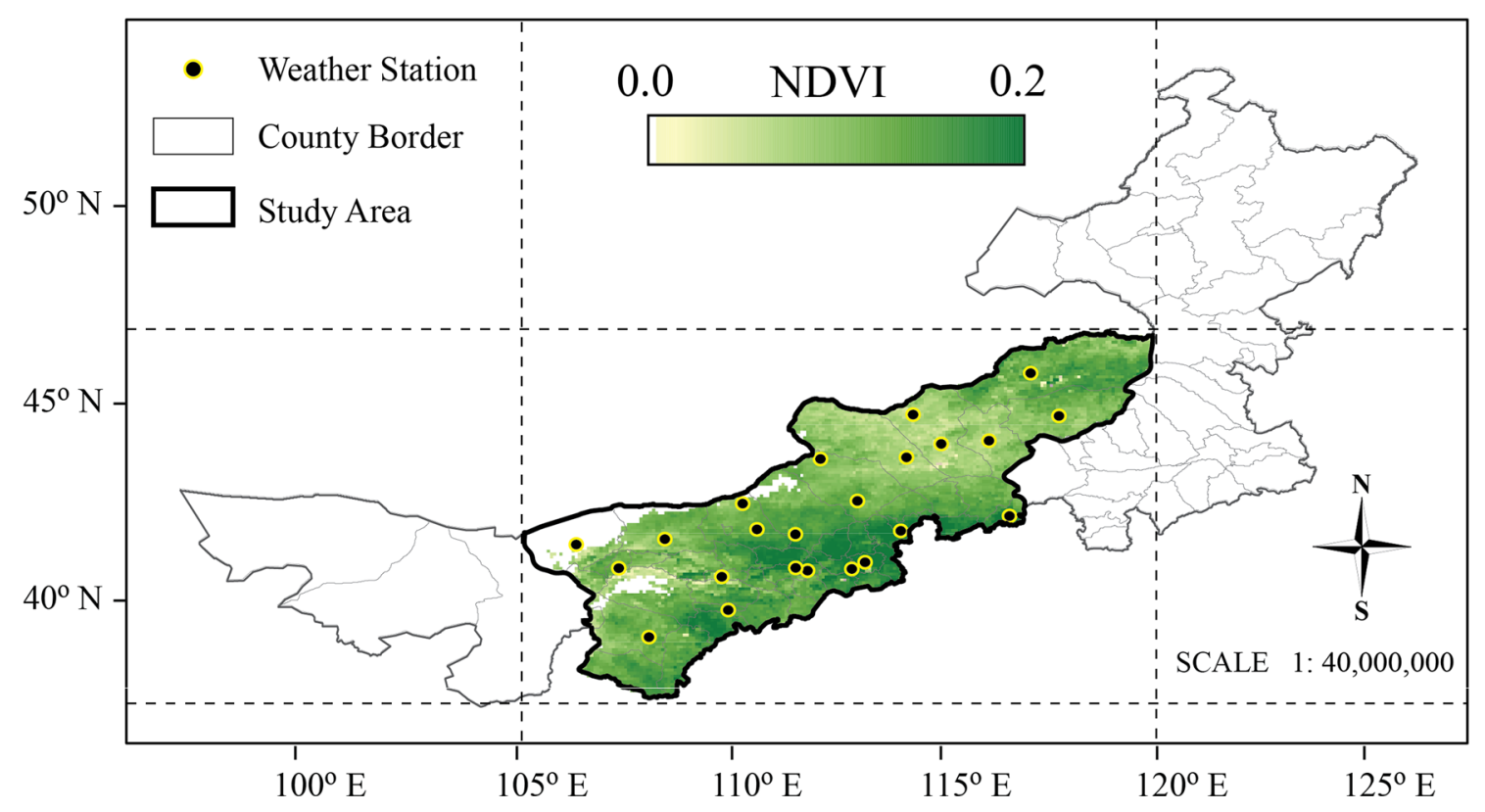

Fig. 2 The study area in central Inner Mongolia $\left(37.61^{\circ}-46.78^{\circ} \mathrm{N}\right.$, $105.24^{\circ}-119.89^{\circ}$ E). Black dots indicate the 23 meteorological stations within the study area, where dust storm observations and associated meteorological data used in this study were recorded. The study area is primarily covered by grassland, semidesert shrubs, and desert (Liu et al. 2013). The spring (February-June) normalized difference vegetation index (NDVI) (shown in green) for the study area was averaged from 1982 to 2008 by using the global inventory modeling and mapping studies (GIMMS) normalized difference vegetation index (NDVI) product derived from observations of the advanced very high resolution radiometer (AVHRR) instruments onboard the National Oceanic and Atmospheric Administration (NOAA) satellites (Tucker et al. 2005) 
http://cdc.cma.gov.cn. The dataset defines severe dust storms by visibility $(\leq 200 \mathrm{~m})$ and instantaneous extreme wind speed $(\geq 20 \mathrm{~m} / \mathrm{s})$.

Annual and decadal variations of severe spring dust storms are shown in Fig. 3. Over the study period, a total of 8451 severe spring dust storms were observed at the 23 ground stations, with an average of 156.5 severe spring dust storms per year. The maximum number of severe spring dust storms (566) was recorded in 1966, and the lowest number was observed in 1997 (Fig. 3). At the decadal scale, the 1960s experienced the highest number of severe spring dust storms (annual average 232.4), and severe spring dust storm occurrence was most restrained in the 1990s (annual average 64.1) (Fig. 3). A significant $(p<0.01)$ linearly decreasing trend of annual spring dust storms indicates a decrease of 2.99 severe spring dust storm events per year from 1954 to 2007.

Using the detailed dust storm observations archived in the SYNOP (surface synoptic observations) reports from the 23 ground stations (Fig. 2), we examined the temporal dynamics of four dust storm types-floating dust, blowing sand, moderate dust storm, and severe dust storm-from 2001 to 2012. The SYNOP reports were downloaded from the China Meteorological Data Sharing Service System. The weather conditions associated with a dust storm event are included in the SYNOP weather reports and are coded. Different types of dust storm events are defined as: floating dust (airborne dust particles are uplifted in the air when a dust storm event has occurred and can reduce horizontal visibility to $<10 \mathrm{~km}$; SYNOP code 06); blowing sand (winds carrying large amounts of dust and sand can reduce horizontal visibility to $1-10 \mathrm{~km}$; SYNOP code 07-08); moderate dust storm (strong winds carrying large amounts of dust and sand can reduce horizontal visibility to $<1 \mathrm{~km}$; SYNOP code 09, 30-32, and 98); and severe dust storm (strong winds carrying large amounts of dust and sand can reduce horizontal visibility to $<500 \mathrm{~m}$; SYNOP code 33-35) (Lim and Chun 2006). Figure 4 shows similar temporal variations for different dust storm types as Fig. 3. The 2001-2012 period shows a declining trend in spring dust storms for each dust storm type. The results in Figs. 3 and 4 together demonstrate the decreasing trend in spring dust storm frequency in northern China since the middle of last century, regardless of dust storm type.

This declining trend of dust storms could be attributed to: (1) changes in the atmospheric circulation background (for example, meridional temperature gradients, cyclone frequencies, and air pressure index) (Ding et al. 2005), (2) changes in near-surface meteorological elements (for example, surface wind and precipitation) (Kurosaki and

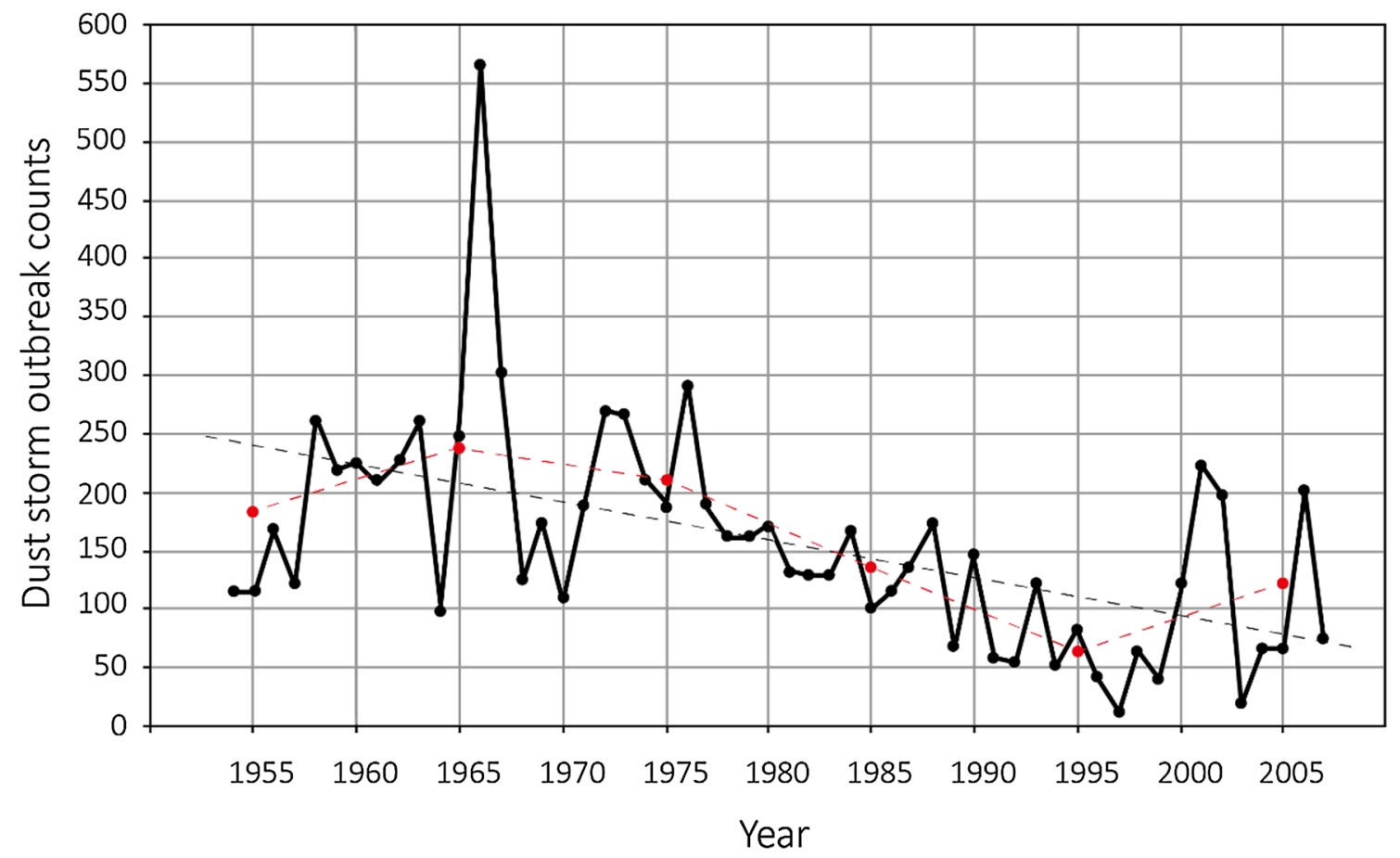

Fig. 3 Long-term temporal dynamics of severe regional spring (February-June) dust storms in central Inner Mongolia from 1954 to 2007 , based on field observations from 23 ground stations. The black line and the red dashed line indicate the annual and decadal

variations of severe spring dust storm counts. The grey dashed line indicates the linear decreasing trend of severe spring dust storms, regressed by using the annual severe spring dust storm outbreaks 

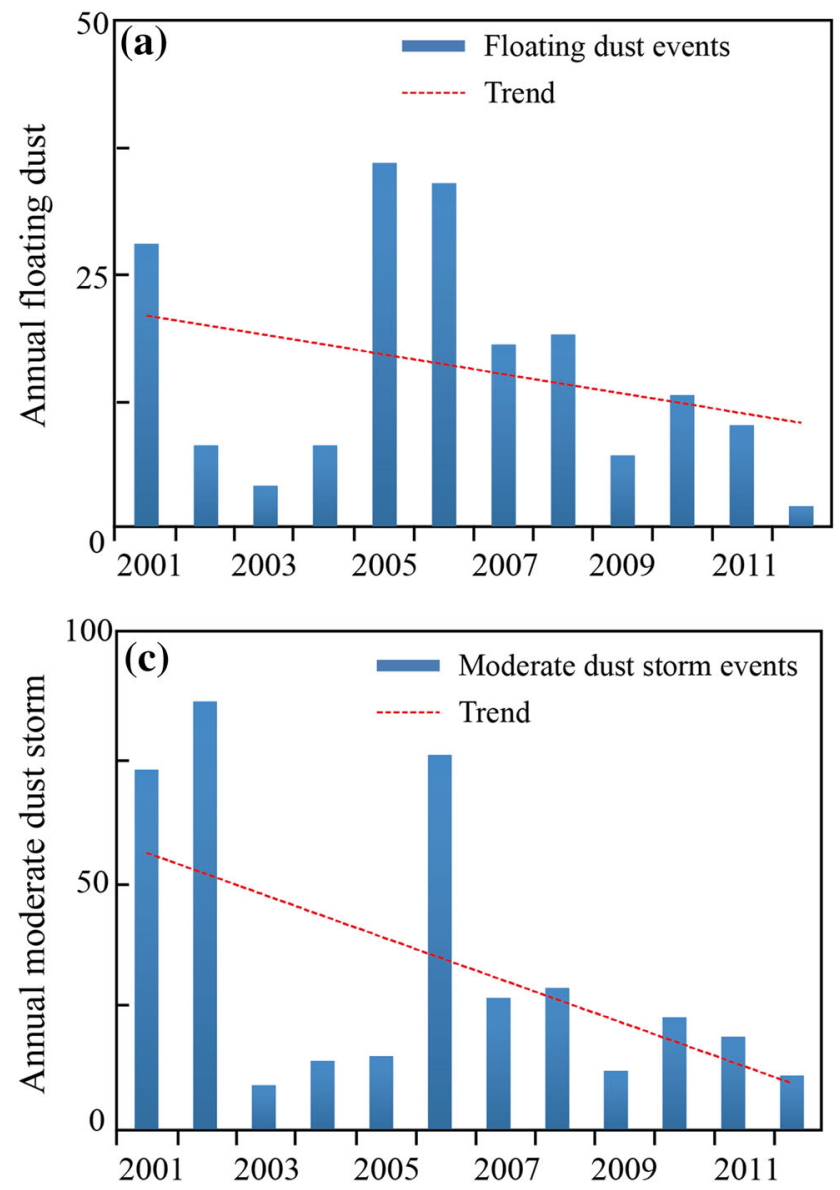

Fig. 4 Temporal dynamics and trends of different types of spring (February-June) dust storms in central Inner Mongolia from 2001 to 2012. Dust storm data are from the SYNOP (surface synoptic

Mikami 2003), and (3) changes in ground roughness (for example, vegetation coverage and land cover) (Qian et al. 2002; Zou and Zhai 2004; Wang et al. 2006). Because of the heterogeneity in global warming, that is, high latitudes tend to warm faster than low latitudes, the meridional temperature gradient and air pressure gradient are reduced, dampening the activity of atmospheric circulation, resulting in surface wind stilling (Vautard et al. 2010). The increased temperature induced more active photosynthesis of vegetation, accumulating more biomass on the ground, which increased surface roughness (Vautard et al. 2010). The slower surface wind speed and higher surface roughness limited the frequency of dust storms in a warming global climate (Fig. 5).

In the East Asia region, vegetation green-up occurs within the dust storm prevailing season (March-May) (Cong et al. 2012). The earlier vegetation green-up date may be another cause of the reduced dust storm frequency under warmer temperatures. Although the restraining effect of vegetation on dust storm outbreaks has been recognized in some studies (Sun et al. 2001; Engelstädter et al. 2003), only the spatial
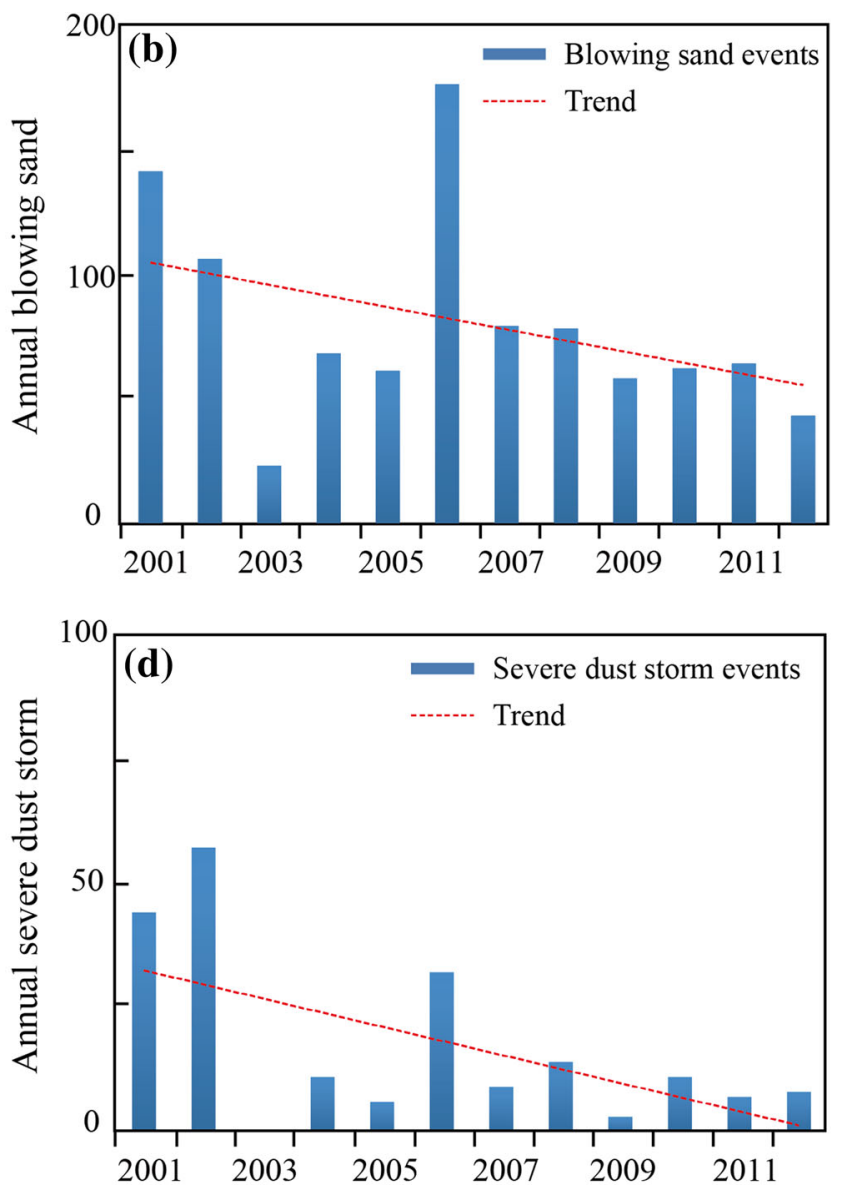

observations) reports from 23 ground stations. Histograms indicate the annual counts of spring dust storms. The red lines indicate the temporal trends of spring dust storms from 2001 to 2012

aspects of vegetation impacts on dust storm frequency (such as vegetation coverage and biomass) were considered, drawing the general conclusion that the more extensive the vegetation coverage and/or the better the vegetation growth, the fewer the dust storms (Kimura 2012; Lee and Kim 2012). However, the potential effects of a shift in vegetation phenology - that is, the timing of the onset of plant regeneration in the spring season and its relation to changes in the seasonal cycles of the climate-on dust storms were ignored (Fan et al. 2014). It is possible that in addition to vegetation coverage (a spatial index), vegetation green-up onset (a temporal index) also affects dust storm outbreaks.

\section{Understanding the Reduced Spring Dust Storm Frequency from the Shift in Vegetation Phenology}

From the temporal point of view, the same amount of vegetation is likely to exert different influences on dust storms when vegetation growing time shifts. When the 
Fig. 5 Reduced dust storm frequency in a warming global climate. Heterogeneous global warming reduces the air pressure gradient and induces surface wind stilling. Increased temperatures enhance photosynthesis, leading to more biomass accumulation, which increases surface roughness and the wind speed needed to blow up dust materials. In the East Asia region, vegetation greenup occurs within the dust storm prevailing season (MarchMay). A shift in vegetation green-up date may be another cause of the change in the dynamics of spring dust storms under warmer temperatures

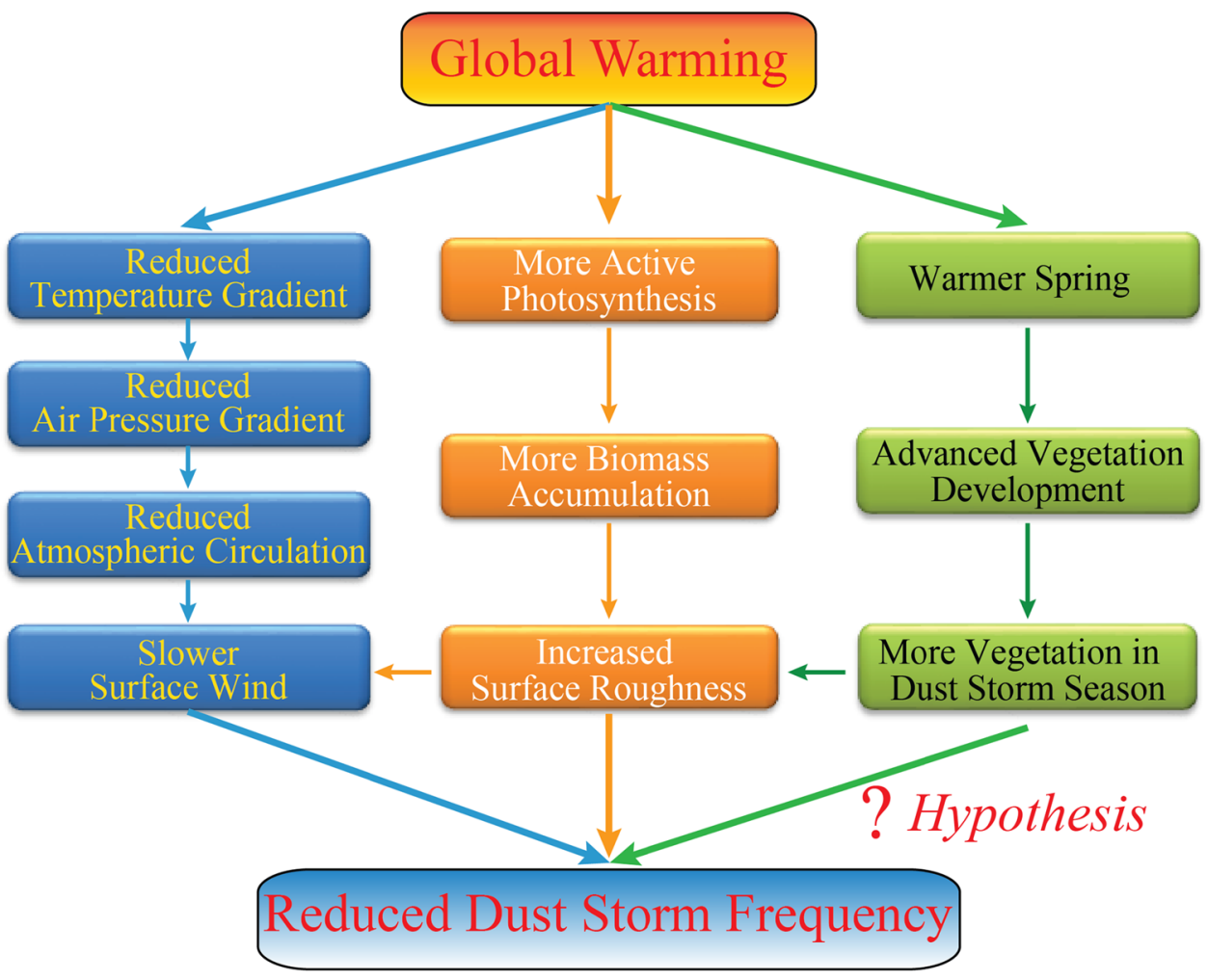

vegetation growing season lags behind the dust storm prevailing season, impacts from vegetation on dust storms weaken, regardless of how well the vegetation grows. Both vegetation amount and growing time might affect dust storm activity. In East Asia, dust storms prevail from March to May, and the vegetation growing season generally lasts from April to October (Jeong et al. 2011). The effective time span for studying the relationship between vegetation and dust storms is between April and May, when the dust storm season and the vegetation growing season overlap.

In previous studies (Lee and Sohn 2011), the average growing season NDVI was usually used to indicate the vegetation condition during the dust storm season. The average growing season NDVI is primarily determined by vegetation growth in summer, when vegetation greenness reaches its maximum. The variation of the NDVI during the dust storm season has limited influence on the average growing season NDVI. The average spring NDVI is dampened by the poor vegetation conditions before the green-up date when plant leaves begin to turn green after winter dormancy, which fails to represent the actual vegetation conditions during the dust storm season. Both the average growing season NDVI and the average spring NDVI are not temporally matched with the dust storm prevailing season in East Asia. Therefore, the average growing season/spring NDVI could not accurately characterize the restrictive effects of vegetation growth on spring dust storms (Fan et al. 2014).

To better assess the impact of vegetation on dust storm occurrence, this study uses the green-up date (occurring in the dust storm season) as an indicator of vegetation condition. The green-up date represents the end of the soil exposure period and the start of rapid vegetation growth. Compared to the average growing season/spring NDVI, the green-up date is more effective to represent the vegetation dynamics during the dust storm season and is better at characterizing the restraining effects of the vegetation on dust storms (Fan et al. 2014). Vegetation green-up dates from 1982 to 2008 were determined by tracing the temporal changes of the satellite-derived vegetation index (NDVI). We first calculated the multiyear (1982-2008) average annual NDVI time series using the original 15-day resolution NDVI data. The NDVI ratio $\left(\mathrm{NDVI}_{\text {ratio }}\right)$ was defined as (Piao et al. 2006):

$\operatorname{NDVI}_{\text {ratio }}(t)=[\operatorname{NDVI}(t+1)-\operatorname{NDVI}(t)] /[\operatorname{NDVI}(t)]$,

where $t$ is the day of the year (at a 15-day resolution). We then located the date when the $\mathrm{NDVI}_{\text {ratio }}$ reached the maximum, and used the corresponding $\operatorname{NDVI}(t)$ value as the threshold.

The annual vegetation green-up date was calculated as the day of a study year when the corrected (anomalies 
caused by clouds, snow cover, orbital drifts and volcanic stratospheric aerosol) interpolated 1-day resolution NDVI crossed the threshold value. If the maximum NDVI value of a certain pixel in a year was smaller than 0.2 , this pixel was considered bare land in this year and excluded from green-up date identification. The NDVI time series used in this study are from the global inventory monitoring and modeling studies (GIMMSs) database obtained from the advanced very high resolution radiometer (AVHRR) remote-sensed observations (Tucker et al. 2005). Specific descriptions of vegetation green-up date extractions from NDVI time series can be found in Fan et al. (2014).

Figure 6 shows the spring dust storm outbreaks and pixel-scale vegetation green-up date anomalies of the study area in 1997, 2004, and 2006. These 3 years were selected to represent recent extremely weak (1997), medium (2004), and extremely strong (2006) dust storm years. In 1997, only 21 dust storm events were observed in the spring (February-June) in central Inner Mongolia, and $73 \%$ of the study area had an advanced green-up date (Fig. 6a). In the medium dust storm year (2004), 101 spring dust storms were recorded, and the satellite-derived area with earlier vegetation green-up was $42 \%$ of the study area (Fig. 6b). However, in the extremely strong dust storm year (2006), when satellite data suggest that only $18 \%$ of the study area showed advanced vegetation spring phenology, 319 spring dust storms were observed (Fig. 6c). Figure 6 indicates a clear dampening effect of earlier vegetation green-up on dust storm frequency.

Generally, after green-up, vegetation grows rapidly and forms a more complex canopy structure (Bazzaz 1979; Zhang et al. 2003). A wind slowdown area appears behind plants and friction velocity declines. Higher vegetation coverage after green-up also increases surface roughness, raising the threshold wind speed for initiating a dust storm and thus reducing the possibility of a dust storm outbreak (Kimura 2012; Lee and Kim 2012). Due to better vegetation coverage, soil surface moisture may increase by hydraulic lift, when plant roots absorb water from deep soils (Dawson 1993). Greater vegetation coverage also leads to less surface runoff, which further increases surface soil moisture content and increases adsorption between soil particles and forms soil aggregates. Soil aggregates decrease the susceptibility of the land surface to strong winds (Nickling and Neuman 2009). Additionally, an earlier vegetation green-up pushes forward the end of the soil exposure period. Even when considering the duration of high soil surface exposure remains unchanged, with earlier green-up the soil exposure period that occurs earlier in the spring season corresponds to a time period with less strong wind events, thus resulting in a decrease in total spring dust storms (Fan et al. 2014). If the shortening of the soil exposure period due to earlier green-up is taken into
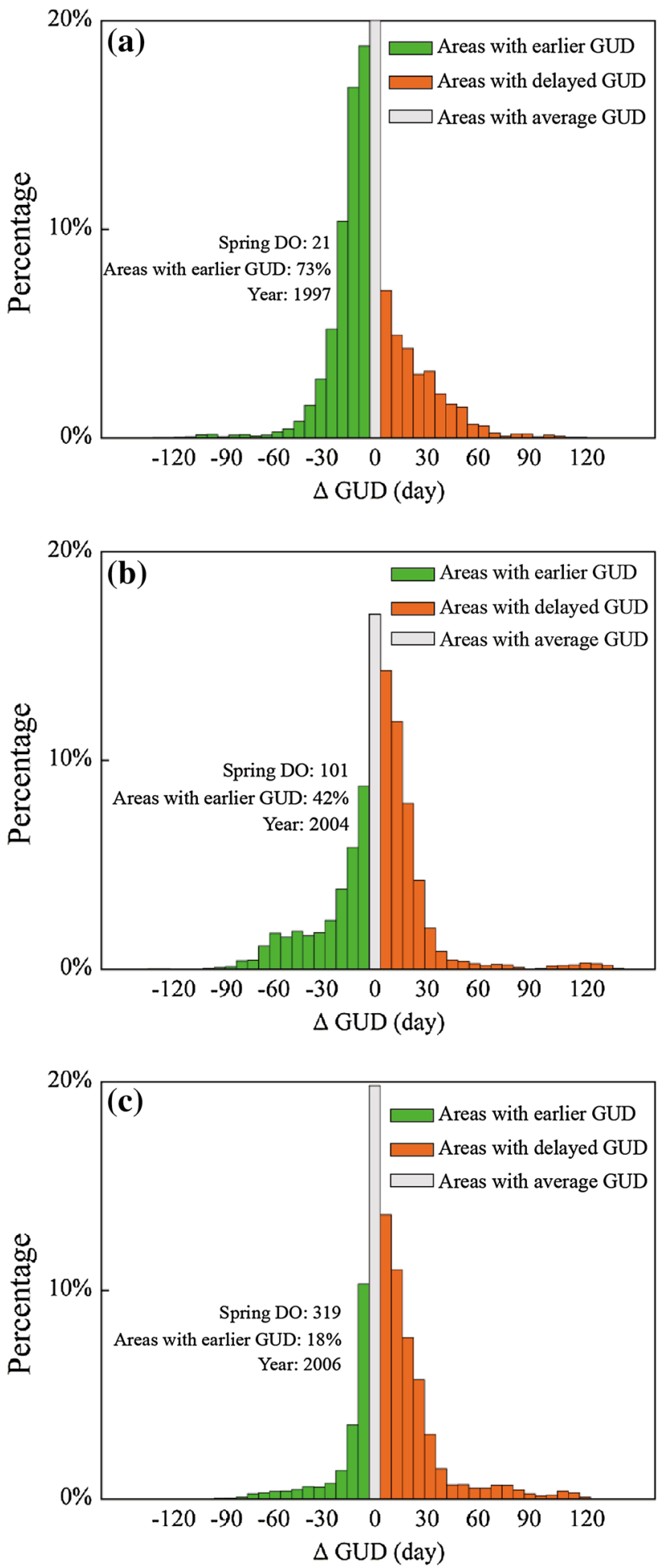

Fig. 6 The dampening effect of an earlier vegetation green-up date (GUD) on spring dust storm frequency in central Inner Mongolia, showing the dust storm outbreaks (DO) and pixel-scale $\left(8 \times 8 \mathrm{~km}^{2}\right)$ GUD anomalies ( $\triangle$ GUD) in a an extremely weak dust storm year (1997), b a medium dust storm year (2004), and c an extremely strong dust storm year (2006). Histograms display the percentage of pixels within the study area with earlier GUD (indicated by green bars), average GUD (indicated by grey bars), and delayed GUD (indicated by orange bars) 
Fig. 7 Possible mechanisms for the reduced spring dust storm frequency in central Inner Mongolia caused by earlier vegetation growth in the spring. Due to earlier green-up, vegetation coverage increases and canopy structure becomes more complex, leading to a greater restrictive effect of the vegetation on surface wind speed in the dust storm season. Together with improved surface soil moisture conditions, the threshold wind speed needed to blow up surface dust materials is also increased. Therefore, spring dust storm frequency declines in years with earlier vegetation green-up dates (GUDs)

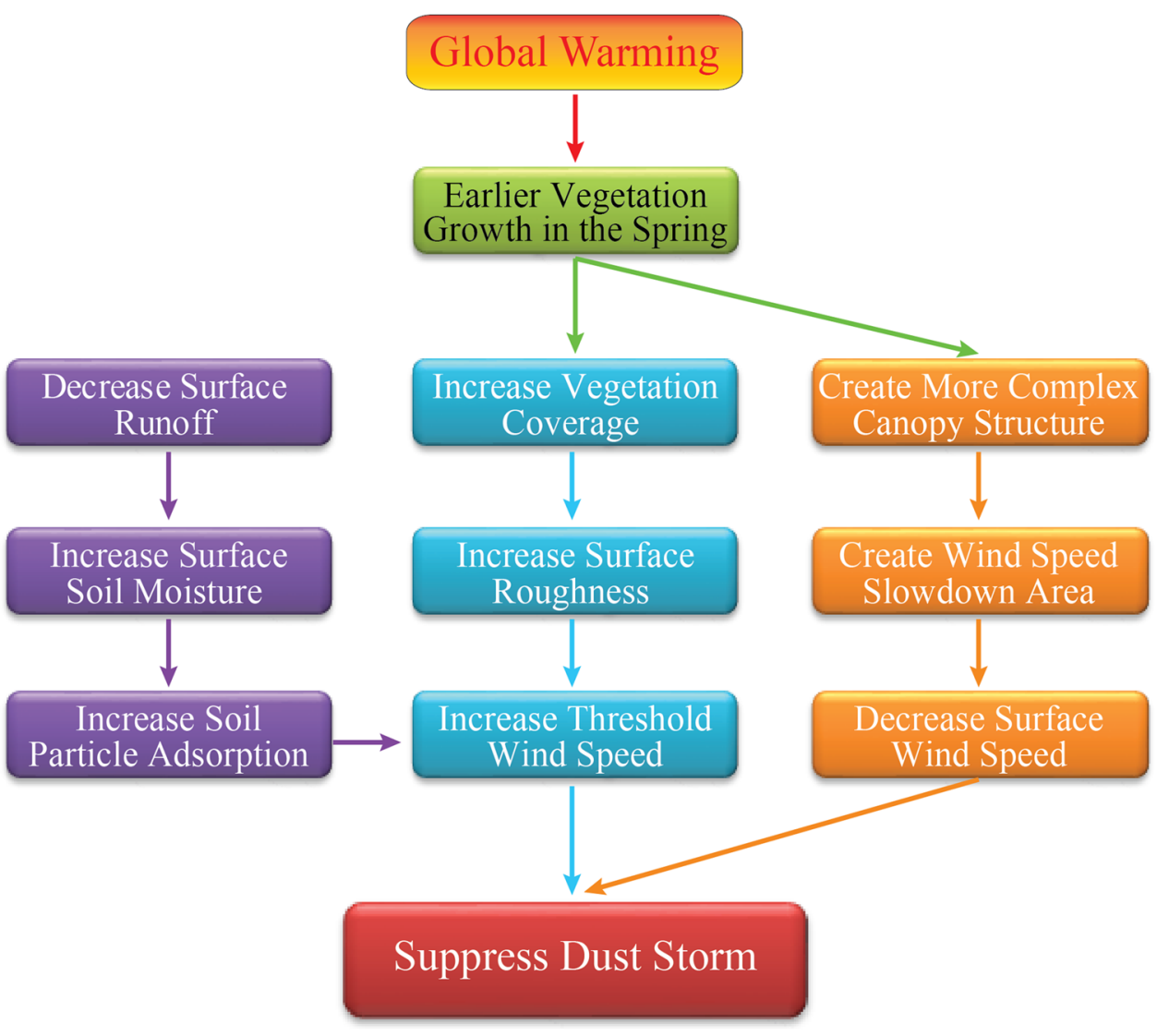

account, the restraining effect of earlier vegetation growth on spring dust storms is more significant. Therefore, after vegetation green-up, dust storms decrease (Fan et al. 2014) (Fig. 7).

Among the different influencing factors of dust storm emergence, such as gust, sediment supply, and ground roughness, the condition of the vegetation is just one factor determining the frequency of dust storms (Fan et al. 2014). Hydrothermal conditions are the ultimate factor influencing dust storm outbreaks. However, the relationship between hydrothermal conditions and dust storms is not simply linear (Fan et al. 2014). No significant correlation was found between spring temperature/precipitation and spring dust storm occurrence in previous studies (Qian et al. 2002; Zou and Zhai 2004). Vegetation green-up is an integrated indicator of the hydrothermal conditions of the plant growth environment. Suitable conditions for vegetation green-up - a warm spring with enough precipitation-are adverse conditions for dust storm outbreaks. Unlike the nonlinear relationship between dust storms and hydrothermal conditions, a linear correlation can be established between vegetation green-up date and dust storms (Fan et al. 2014). Because vegetation green-up is a synthetical expression of hydrothermal conditions, there is a significant impact from the shift in vegetation green-up on dust storm outbreaks. Therefore, variations in vegetation spring phenology (indicating a balance between overall local hydrothermal conditions) supplement the understanding of the dynamics of spring dust storms in East Asia in the changing climate.

\section{Conclusion}

Based on the ground observations of dust storm events in central Inner Mongolia, we demonstrated a significant declining trend of severe spring dust storms from 1954 to 2007. Four types of dust storm events displayed similar decreasing trends in the spring from 2001 to 2012. This kind of reduced spring dust storm frequency in East Asia has been attributed to changes in atmospheric circulation background, near-surface meteorological elements, and ground roughness under the warmer temperatures. This study used percentages of the study area with advanced vegetation green-up onset as an explanatory factor to explain the variations in spring dust storm occurrences. We demonstrated a dampening effect of earlier vegetation growth in the spring on regional spring dust storm frequency. 
Acknowledgments This study was supported by the National Natural Science Foundation of China (41171401) and the National Basic Research Program of China (973) (2012CB955402) Programs. The authors would like to acknowledge the anonymous reviewers for their useful comments, which have greatly helped improve the manuscript.

Open Access This article is distributed under the terms of the Creative Commons Attribution 4.0 International License (http://crea tivecommons.org/licenses/by/4.0/), which permits unrestricted use, distribution, and reproduction in any medium, provided you give appropriate credit to the original author(s) and the source, provide a link to the Creative Commons license, and indicate if changes were made.

\section{References}

Bazzaz, F.A. 1979. The physiological ecology of plant succession. Annual Review of Ecology and Systematics 10: 351-371.

Catt, J.A. 1988. Loess - Its formation, transport and economic significance. In Physical and chemical weathering in geochemical cycles, ed. A. Lerman, and M. Meybeck, 113-142. Dordrecht: Kluwer Academic.

Chen, K.Y. 2010. The northern path of Asian dust transport from the Gobi desert to North America. Atmospheric and Oceanic Science Letters 3(3): 155-159.

Cong, N., S. Piao, A. Chen, X. Wang, X. Lin, S. Chen, S. Han, G. Zhou, and X. Zhang. 2012. Spring vegetation green-up date in China inferred from SPOT NDVI data: A multiple model analysis. Agricultural and Forest Meteorology 165: 104-113.

Cowie, S.M., P. Knippertz, and J.H. Marsham. 2013. Are vegetationrelated roughness changes the cause of the recent decrease in dust emission from the Sahel? Geophysical Research Letters 40(9): 1868-1872.

Creamean, J.M., K.J. Suski, D. Rosenfeld, A. Cazorla, P.J. DeMott, R.C. Sullivan, A.B. White, F.M. Ralph, et al. 2013. Dust and biological aerosols from the Sahara and Asia influence precipitation in the western US. Science 339(6127): 1572-1578.

Dawson, T.E. 1993. Hydraulic lift and water use by plants: Implications for water balance, performance and plant-plant interactions. Oecologia 95(4): 565-574.

Ding, R.Q., J.P. Li, S.G. Wang, and F.M. Ren. 2005. Decadal change of the spring dust storm in northwest China and the associated atmospheric circulation. Geophysical Research Letters 32(2). doi:10.1029/2004GL021561.

Ebata, M., and R. Tateishi. 2001. Phenological stage monitoring in Siberia using NOAA/AVHRR data. Paper presented at the 22nd Asian Conference on Remote Sensing, 5-9 November 2001, Singapore, Centre for Remote Imaging, Sensing and Processing (CRISP).

Engelstädter, S., K.E. Kohfeld, I. Tegen, and S.P. Harrison. 2003. Controls of dust emissions by vegetation and topographic depressions: An evaluation using dust storm frequency data. Geophysical Research Letters 30(6). doi:10.1029/ 2002GL016471.

Fan, B.H., L. Guo, N. Li, J. Chen, H. Lin, X.Y. Zhang, M.G. Shen, Yuhan Rao, et al. 2014. Earlier vegetation green-up has reduced spring dust storms. Scientific Reports 4. doi:10.1038/srep06749.

Goudie, A.S. 1983. Dust storms in space and time. Progress in Physical Geography 7(4): 502-530.

Goudie, A.S. 2009. Dust storms: Recent developments. Journal of Environmental Management 90(1): 89-94.

Goudie, A.S., and N.J. Middleton. 1992. The changing frequency of dust storms through time. Climatic Change 20(3): 197-225.
Griffin, D.W., and C.A. Kellogg. 2004. Dust storms and their impact on ocean and human health: Dust in Earth's atmosphere. EcoHealth 1(3): 284-295.

Idso, S.B. 1974. Thermal blanketing: A case for aerosol-induced climatic alteration. Science 186(4158): 50-51.

Jeong, S.J., C.H. Ho, H.J. Gim, and M.E. Brown. 2011. Phenology shifts at start vs. end of growing season in temperate vegetation over the Northern Hemisphere for the period 1982-2008. Global Change Biology 17(7): 2385-2399.

Kimura, R. 2012. Factors contributing to dust storms in source regions producing the yellow-sand phenomena observed in Japan from 1993 to 2002. Journal of Arid Environments 80: 40-44.

Kurosaki, Y., and M. Mikami. 2003. Recent frequent dust events and their relation to surface wind in East Asia. Geophysical Research Letters 30(14). doi:10.1029/2003GL017261.

Kurosaki, Y., M. Shinoda, M. Mikami, and B. Nandintsetseg. 2011. Effects of soil and land surface conditions in summer on dust outbreaks in the following spring in a Mongolian grassland. SOLA 7: 69-72.

Lee, J.J., and C.H. Kim. 2012. Roles of surface wind, NDVI and snow cover in the recent changes in Asian dust storm occurrence frequency. Atmospheric Environment 59: 366-375.

Lee, E.H., and B.J. Sohn. 2011. Recent increasing trend in dust frequency over Mongolia and Inner Mongolia regions and its association with climate and surface condition change. Atmospheric Environment 45(27): 4611-4616.

Li, H., X. Yang, Y. Zhao, M. Wang, and W. Huo. 2014. The atmospheric circulation patterns influencing the frequency of spring sand-dust storms in the Tarim Basin. Sciences in Cold and Arid Regions 6(2): 168-173.

Lim, J.Y., and Y. Chun. 2006. The characteristics of Asian dust events in Northeast Asia during the springtime from 1993 to 2004. Global and Planetary Change 52(1-4): 231-247.

Liu, L.Y., P.J. Shi, X. Hu, T.K. Liu, L.L. Guo, X.X. Zhang, Y. Tang, Y.L. Lv, et al. 2011. Natural factors influencing blown sand hazards in Beijing. International Journal of Disaster Risk Science 2(2): 23-31.

Liu, H., F. Tian, H.C. Hu, H.P. Hu, and M. Sivapalan. 2013. Soil moisture controls on patterns of grass green-up in Inner Mongolia: An index based approach. Hydrology and Earth System Sciences 17(2): 805-815.

Mao, R., C.H. Ho, S. Feng, D.Y. Gong, and Y. Shao. 2013. The influence of vegetation variation on Northeast Asian dust activity. Asia-Pacific Journal of Atmospheric Sciences 49(1): 87-94.

Nickling, W.G., and C.M. Neuman. 2009. Aeolian sediment transport. In Geomorphology of desert environments, ed. A.J. Parsons, and A.D. Abrahams, 517-555. New York: Springer.

Piao, S.L., J.Y. Fang, L.M. Zhou, P. Ciais, and B. Zhu. 2006. Variations in satellite-derived phenology in China's temperate vegetation. Global Change Biology 12(4): 672-685.

Qian, W., L. Quan, and S. Shi. 2002. Variations of the dust storm in China and its climatic control. Journal of Climate 15(10): 1216-1229.

Schlesinger, W.H., J.F. Reynolds, G.L. Cunningham, L. Huenneke, W.M. Jarrell, R.A. Virginia, and W.G. Whitford. 1990. Biological feedbacks in global desertification. Science 247(4946): $1043-1048$

Shao, Y., and C.H. Dong. 2006. A review on East Asian dust storm climate, modelling and monitoring. Global and Planetary Change 52(1): 1-22.

Shao, Y., M. Klose, and K.H. Wyrwoll. 2013. Recent global dust trend and connections to climate forcing. Journal of Geophysical Research: Atmospheres 118(19): 11107-11118.

Song, Y., Z.J. Quan, L.Y. Liu, P. Yan, and T. Cao. 2005. The influence of different underlying surface on sand-dust storm in 
northern China. Journal of Geographical Sciences 15(4): 431-438.

Sun, J., M. Zhang, and T. Liu. 2001. Spatial and temporal characteristics of dust storms in China and its surrounding regions, 1960-1999: Relations to source area and climate. Journal of Geophysical Research: Atmospheres 106(D10): 10325-10333.

Tan, M., X. Li, and L. Xin. 2014. Intensity of dust storms in China from 1980 to 2007: A new definition. Atmospheric Environment 85: $215-222$.

Toon, O.B. 2003. Atmospheric science: African dust in Florida clouds. Nature 424(6949): 623-624.

Tucker, C.J., J.E. Pinzon, M.E. Brown, D.A. Slayback, E.W. Pak, R. Mahoney, E.F. Vermote, and N. El Saleous. 2005. An extended AVHRR 8-km NDVI dataset compatible with MODIS and SPOT vegetation NDVI data. International Journal of Remote Sensing 26(20): 4485-4498.

Vautard, R., J. Cattiaux, P. Yiou, J.N. Thepaut, and P. Ciais. 2010. Northern Hemisphere atmospheric stilling partly attributed to an increase in surface roughness. Nature Geoscience 3(11): $756-761$.

Wang, S.G., D.B. Yang, Y.S. Zhou, T. Ba, and C.L. Xin. 1995. Analysis on the formative causes of sand-dust storms in the Northwest China during 3-12 April 1994. Journal of Desert Research 15(4): 332-338 (in Chinese).

Wang, X., Z. Dong, J. Zhang, and L. Liu. 2004. Modern dust storms in China: An overview. Journal of Arid Environments 58(4): 559-574.
Wang, X., Z. Zhou, and Z. Dong. 2006. Control of dust emissions by geomorphic conditions, wind environments and land use in northern China: An examination based on dust storm frequency from 1960 to 2003. Geomorphology 81(3-4): 292-308.

Xu, Q.Y., and J.S. Hu. 1997. Spatial-temporal characteristic analysis of dust weather in northwestern China. In Study on the duststorm in China, ed. Z.Y. Fang, 11-15. Beijing: China Meteorological Press (in Chinese).

Yang, Y.Q., Q. Hou, C.H. Zhou, H.L. Liu, Y.Q. Wang, and T. Niu. 2008. Sand/dust storm processes in Northeast Asia and associated large-scale circulations. Atmospheric Chemistry and Physics 8(1): 25-33.

Zhang, X.Y., R. Arimoto, and Z.S. An. 1997. Dust emission from Chinese desert sources linked to variations in atmospheric circulation. Journal of Geophysical Research: Atmospheres 102(D23): 28041-28047.

Zhang, X., M.A. Friedl, C.B. Schaaf, A.H. Strahler, J.C.F. Hodges, F. Gao, B.C. Reed, and A. Huete. 2003. Monitoring vegetation phenology using MODIS. Remote Sensing of Environment 84: 471-475.

Zhou, L.M., C.J. Tucker, R.K. Kaufmann, D. Slayback, N.V. Shabanov, and R.B. Myneni. 2001. Variation in northern vegetation activity inferred from satellite data of vegetation index during 1981 to 1999. Journal of Geophysical Research 106(D17): 20069-20083.

Zou, X.K., and P.M. Zhai. 2004. Relationship between vegetation coverage and spring dust storms over northern China. Journal of Geophysical Research: Atmospheres 109(D3). doi:10.1029/ 2003JD003913. 